\title{
Quantum information-based analysis of electron-deficient bonds
}

Cite as: J. Chem. Phys. 150, 204117 (2019); https://doi.org/10.1063/1.5093497

Submitted: 21 February 2019 . Accepted: 06 May 2019 . Published Online: 30 May 2019

Jan Brandejs (D), Libor Veis (D), Szilárd Szalay, Gergely Barcza, Jiří Pittner, and Örs Legeza

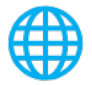

\section{ARTICLES YOU MAY BE INTERESTED IN}

Range-separated hybrid density functionals made simple

The Journal of Chemical Physics 150, 201102 (2019); https://doi.org/10.1063/1.5097164

An alternative derivation of orbital-free density functional theory

The Journal of Chemical Physics 150, 204109 (2019); https://doi.org/10.1063/1.5096405

Performance of new density functionals of nondynamic correlation on chemical properties

The Journal of Chemical Physics 150, 204101 (2019); https://doi.org/10.1063/1.5082745

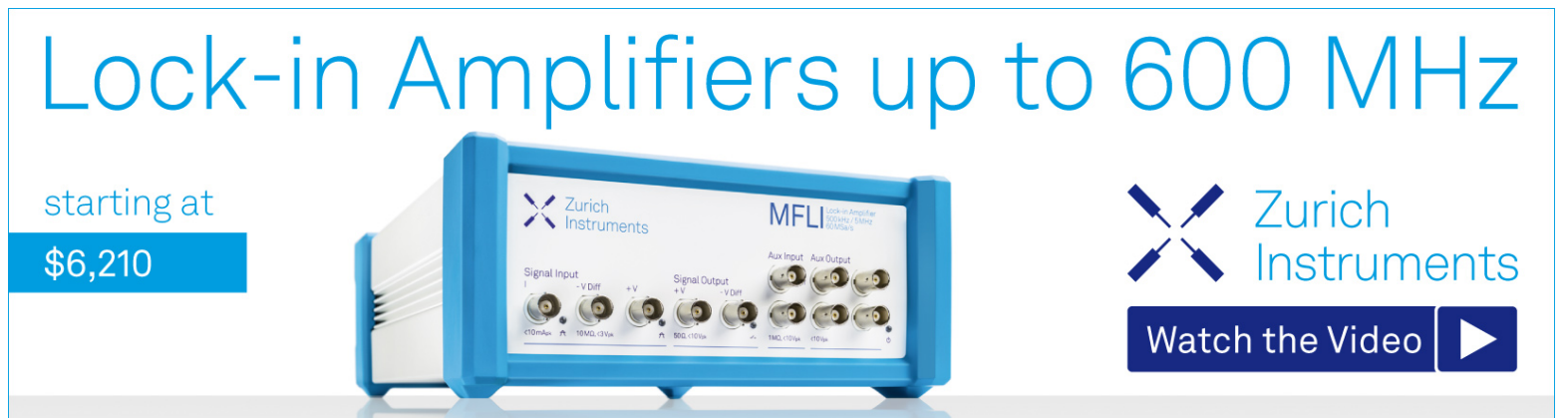




\title{
Quantum information-based analysis of electron-deficient bonds
}

\author{
Cite as: J. Chem. Phys. 150, 204117 (2019); doi: 10.1063/1.5093497 \\ Submitted: 21 February 2019 - Accepted: 6 May 2019 • \\ Published Online: 30 May 2019

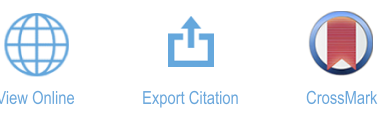

Jan Brandejs, ${ }^{1,2, a)}$ (D) Libor Veis, ${ }^{1, b)}$ (D) Szilárd Szalay, ${ }^{3, c)}$ Gergely Barcza, ${ }^{1,3, d)}$ Jiří Pittner, $^{1, e)}$ and Örs Legeza ${ }^{3, f)}$

\begin{abstract}
AFFILIATIONS 18223 Prague 8, Czech Republic

a) Electronic mail: jan.brandejs@jh-inst.cas.cz

b) Electronic mail: libor.veis@jh-inst.cas.cz

${ }^{c}$ Electronic mail: szalay.szilard@wigner.mta.hu

d) Electronic mail: barcza.gergely@wigner.mta.hu

e) Electronic mail: jiri.pittner@jh-inst.cas.cz

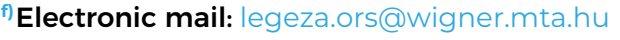

' J. Heyrovský Institute of Physical Chemistry, Academy of Sciences of the Czech Republic, v.v.i., Dolejškova 3,

${ }^{2}$ Faculty of Mathematics and Physics, Charles University, Prague, Czech Republic

${ }^{3}$ Strongly Correlated Systems "Lendület" Research Group, Institute for Solid State Physics and Optics, MTA Wigner Research Centre for Physics, Konkoly-Thege Miklós út 29-33, H-1121 Budapest, Hungary

\begin{abstract}
Recently, the correlation theory of the chemical bond was developed, which applies concepts of quantum information theory for the characterization of chemical bonds, based on the multiorbital correlations within the molecule. Here, for the first time, we extend the use of this mathematical toolbox for the description of electron-deficient bonds. We start by verifying the theory on the textbook example of a molecule with three-center two-electron bonds, namely, diborane(6). We then show that the correlation theory of the chemical bond is able to properly describe the bonding situation in more exotic molecules which have been synthesized and characterized only recently, in particular, the diborane molecule with four hydrogen atoms [diborane(4)] and a neutral zerovalent s-block beryllium complex, whose surprising stability was attributed to a strong three-center two-electron $\pi$ bond stretching across the $\mathrm{C}-\mathrm{Be}-\mathrm{C}$ core. Our approach is of high importance especially in the light of a constant chase after novel compounds with extraordinary properties where the bonding is expected to be unusual.
\end{abstract}

Published under license by AIP Publishing. https://doi.org/10.1063/1.5093497

\section{INTRODUCTION}

Recent years have witnessed remarkable interest in application of tools of quantum information theory in chemistry. As a prominent example, the performance of state-of-the-art tensor product methods for electronic structure calculations ${ }^{17,28-34}$ heavily relies on proper manipulation of entanglement. ${ }^{1,4,6,12,14,17,22}$ These include the density matrix renormalization group (DMRG) method, ${ }^{35,36}$ which variationally optimizes wave functions in the form of matrix product states (MPS).

Other important examples represent characterization of electron correlation into its static (strong) and dynamic contributions, automatic (black-box) selection of the active spaces, ${ }^{1,6,17,23,24,38}$ or the self-adaptive tensor network states with multisite correlators, ${ }^{25}$ all of which harness single- and two-orbital entanglement entropies. Last but not least, correlation measures based on the single- and two-orbital entanglement entropies have also been employed for the purposes of bond analysis. ${ }^{10,}$

In the preceding work, ${ }^{26}$ we have presented the very general correlation theory of the chemical bond based on multiorbital correlation measures which goes beyond the scope of two-orbital picture. It is able to properly describe multiorbital bonds, and we have demonstrated its performance on a representative set of organic molecules (aliphatic as well as aromatic).

In the present article, we apply this theory to systems with electron-deficient bonds, i.e., to compounds which have too few valence electrons for the connections between atoms to be described as covalent bonds, which have always fascinated chemists. First, we 
apply the theory to the notoriously known textbook example of the diborane $(6)^{39}$ molecule $\left(\mathrm{B}_{2} \mathrm{H}_{6}\right)$ with two-electron three-center bridge bonds and then also to recently characterized diborane $(4)^{40}$ $\left(\mathrm{B}_{2} \mathrm{H}_{4}\right)$ and zero-valent complexes of beryllium. ${ }^{41,42}$ The neutral form of the latter compound exhibits surprising stability, which was attributed to a strong three-center two-electron $\pi$ bond stretching across the $\mathrm{C}-\mathrm{Be}-\mathrm{C}$ core. ${ }^{41}$ Unlike in the previous study, ${ }^{26}$ here, we work in the bigger detail in a sense that we also employ eigenstates of multiorbital reduced density matrices, which give us additional insights into the character of bonding.

The article is organized as follows: in the section titled "Studied Systems", we briefly present the studied systems; in the section titled "Methodology", we review the main concepts of the theory of multiorbital correlations; the section titled "Computational Details" presents the computational details, and the section titled "Results" presents the results of our calculations which are followed by their discussion; and the final section closes with conclusions.

\section{STUDIED SYSTEMS}

\section{Diboranes}

In its ground state, diborane(6) [Fig. 1(a)] adopts its most stable conformation with two bridging $\mathrm{B}-\mathrm{H}-\mathrm{B}$ bonds and four terminal B-H bonds. Its structure was first correctly measured in 1943 from infrared spectra of gaseous samples by an undergraduate student, Longuet-Higgins. ${ }^{43,44}$ Subsequent measurements with electron diffraction confirmed his conclusions, ${ }^{45}$ and X-ray diffraction detected further systems with the bridging hydrogen bond. ${ }^{46}$ The $\mathrm{B}-\mathrm{H}-\mathrm{B}$ bridging was considered an atypical electron-deficient covalent chemical bond. ${ }^{47}$ Diborane(6) is a prominent example of a molecule with three-center two-electron bonds. ${ }^{48}$ As it is a well studied system, we use its B-H-B linkage as a reference to compare with bond strengths and properties of more complex systems featuring three-center two-electron bonds.

According to quantum-chemical calculations, ${ }^{49-54}$ different species of diborane with less than six hydrogen atoms should exist, also featuring the bridging $\mathrm{B}-\mathrm{H}-\mathrm{B}$ bonds. However, all candidates are short-lived reaction intermediates, difficult to prepare and to identify. Hence, no neutral species has been identified experimentally until 2015 when Chou irradiated diborane(6) dispersed in neon at $3 \mathrm{~K}$ with far-ultraviolet light, detecting diborane(4), $\mathrm{B}_{2} \mathrm{H}_{4}$ [Fig. 1(b)]. ${ }^{40}$ This new species with two terminal hydrogen atoms possesses two bridging hydrogen atoms, and so it became the simplest neutral boron hydride identified with such a structural feature.

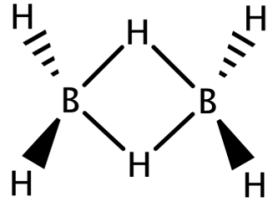

(a)

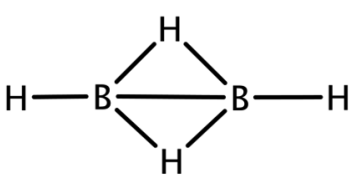

(b)
FIG. 1. Structures of (a) diborane(6) and (b) diborane(4).

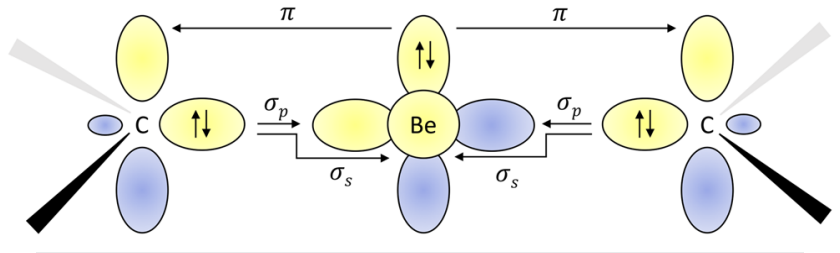

FIG. 2. Schematic representation of the $\mathrm{C}-\mathrm{Be}-\mathrm{C}$ bonding mechanisms.

\section{Beryllium complexes}

Complexes of metal atoms of the s-block of the periodic table are often found in their zero oxidation state due to their exceptional electron donation. For their interesting reactivities, these became frequent synthetic targets, competing with traditional transition metal complexes. ${ }^{55-57}$ We follow the recent experimental work of Arrowsmith, who isolated, for the first time, neutral compounds with zero-valent s-block metal, beryllium. ${ }^{41}$ These brightly colored molecules have very short $\mathrm{Be}-\mathrm{C}$ bonds and beryllium in linear coordination geometries. ${ }^{58-61}$ This indicates strong multiple $\mathrm{Be}-\mathrm{C}$ bonding. According to the theoretical and spectroscopic results, the molecules adopt a closed-shell singlet configuration with a $\operatorname{Be}(0)$ metal center. ${ }^{41}$ The complexes are surprisingly stable, and this was ascribed to an unusually strong three-center two-electron $\pi$ bond stretching across the $\mathrm{C}-\mathrm{Be}-\mathrm{C}$ unit. Two bonding mechanisms depicted in Fig. 2 are taking place, namely, $\sigma$ donation from the carbon doubly occupied $\mathrm{sp}^{2}$ hybrid orbital to empty $\mathrm{s}$ and $\mathrm{p}_{\mathrm{x}}$ orbitals on the central Be atom and $\pi$ back donation from the beryllium $\mathrm{p}_{z}$ orbital to $\mathrm{p}_{z}$ orbitals located on $\mathrm{C}$ atoms.

We studied two of the proposed systems: first, the $\mathrm{Be}(\mathrm{CAC})_{2}$ complex [Fig. 6(a)], where CAC corresponds to cyclic amino carbene donors, which stabilize the compound due to their $\pi$-acidity. ${ }^{62,63}$ We performed a multireference calculation in order to verify the proposed singlet configuration with a $\mathrm{Be}(0)$ metal center and to provide a deeper insight into the bonding scheme. Next, we studied dication $\left[\mathrm{Be}(\mathrm{CAC})_{2}\right]^{2+}[$ Fig. $6(\mathrm{~b})]$, in which the removal of two electrons disrupts the bridging $\mathrm{C}-\mathrm{Be}-\mathrm{C}$ bond. This allowed us to compare with the neutral $\mathrm{Be}(\mathrm{CAC})_{2}$ and to determine the stabilization effect of the bridging bond.

\section{METHODOLOGY}

Recently, the correlation theory of the chemical bond ${ }^{26}$ was developed, characterizing bonds based on the correlations among orbitals localized on individual atoms. Simply put, if we think of a simple covalent bond and localize the bonding and antibonding molecular orbitals (MOs) into their atomic contributions, these localized orbitals will be highly correlated. Therefore, standard twoorbital bonds can be characterized by pairs of strongly correlated localized orbitals, and the strength of the correlation characterizes the strength of the bond from the quantum information theoretical point of view.

The correlation theory of the chemical bond can also be used for the characterization of bonds more involved than the covalent bonds. The concept in general is to find the finest possible correlation based clustering of the localized orbitals into clusters so that the clusters are weakly correlated with each other and the orbitals 
inside the clusters are strongly correlated. ${ }^{26}$ These clusters then form independent bonds of a Lewis structure of a given molecule, and the strength of the correlation with respect to this clustering refers to the validity of such a representation. The weaker the correlation is, the better the Lewis structure represents bonding.

In order to review the correlation measures, ${ }^{64}$ which will be used in our analysis, let us denote the set of (the labels of) localized orbitals with $M$. We aim at investigating the correlations in an $L \subseteq M$ set of orbitals (cluster). The state of the full electronic system of the cluster $L$ is given by the density operator $\varrho_{L}$, while the reduced state of a (sub)cluster $X \subseteq L$ is given by the reduced density operator $\rho_{X}$ in general. ${ }^{65-67}$ If the cluster of orbitals $L$ can be given by a state vector $\left|\psi_{L}\right\rangle$ (for example, when a given eigenstate of the whole molecule is considered), then its density operator is of rank one, $\varrho_{L}=\left|\psi_{L}\right\rangle\left\langle\psi_{L}\right|$, called a pure state. Its reduced density operator is usually mixed (not of rank one), which is the manifestation of entanglement ${ }^{68}$ between the (sub)cluster $X$ and the rest of the cluster $L \backslash X$. In general, a density operator $\varrho_{X}$ can be decomposed in infinitely many ways into state vectors $\left|\psi_{i}\right\rangle$ with mixing weights $p_{i} \geq 0$ as $\varrho_{X}=\sum p_{i}\left|\psi_{i}\right\rangle\left\langle\psi_{i}\right|$. The spectral decomposition (where the weights are the $\lambda_{i}$ eigenvalues, and the $\left|\psi_{i}\right\rangle$-s are eigenvectors, being orthogonal) is a special one, in the sense that its weights are the least mixed. ${ }^{69,70}$ Each eigenvector $\left|\psi_{i}\right\rangle$ can be expanded in the occupation number basis; the square of the absolute value of the coefficients is the weights of the given occupations in that given eigenvector $\left|\psi_{i}\right\rangle$ of weight $\lambda_{i}$.

On the first level, the correlation is defined with respect to a partition ${ }^{71}$ of the $L$ set of the orbitals, ${ }^{26,64,72,73}$ denoted with $\xi=\left\{X_{1}, X_{2}, \ldots, X_{|\xi|}\right\} \equiv X_{1}\left|X_{2}\right| \cdots \mid X_{|\xi|}$, where the clusters $X \in \xi$, called parts, are disjoint subsets of the cluster $L$, and $\cup_{X \in \xi} X=L$. The measure of correlation among the parts $X \in \xi$ is the $\xi$-correlation ${ }^{26,64}$

$$
C(\xi):=\sum_{X \in \xi} S(X)-S(L) .
$$

Here, $S(X)=-\operatorname{tr}\left(\varrho_{X} \log _{4} \varrho_{X}\right)$ is the von Neumann entropy. ${ }^{65,67}$ (Note that we use the logarithm to base 4 , which is the dimension of the Hilbert space of an orbital. The resulting numerical values are then the same as the original measures with natural logarithm ${ }^{26}$ given in the units of $\ln 4$. Note that $S(X) \leq|X|$, where $|X|$ is the number of orbitals in cluster $X$.) As a special case, the correlation of two single orbitals,

$$
C(i \mid j)=S(i)+S(j)-S(i, j)=I(i \mid j)
$$

is the well-known (two-orbital) mutual information, ${ }^{65,67,74}$ which has already been considered in chemistry. 6,10-13,16,18-20,26,75-79 (For convenience, we omit the curly brackets \{\} in the cases when this does not cause confusion.) For a general partition $\xi$, we have the bound $^{26}$

$$
C(X \mid Y) \leq 2\left(|L|-\max _{X \in \xi}|X|\right),
$$

which for a bipartition $\xi=X \mid Y$ reduces to

$$
C(X \mid Y) \leq 2 \min \{|X|,|Y|\} .
$$

Note that $C(\xi)$ is zero for the trivial split $\xi=T=\{L\}$, and it takes its maximum, $C(\perp)$, for the finest split $\xi=\perp=\{\{i\}: i \in L\}$. The latter quantity is also called total correlation ${ }^{2,}$

$$
C_{\mathrm{tot}}(L):=C(\perp)=\sum_{i \in L} S(i)-S(L) .
$$

[Note that if cluster $L$ is described by a pure state, then $S(L)=0$ and the correlation is entirely quantum entanglement. ${ }^{64,68,84}$ Moreover, the correlation in a pure state with respect to a bipartition $\xi=X \mid$ $(L \backslash X)$ is just two times the usual entanglement entropy ${ }^{67,85,86}$

$$
C(X \mid(L \backslash X))=2 S(X)=2 S(L \backslash X)
$$

because of the Schmidt decomposition of pure states. ${ }^{67,86,87}$ ]

On the second level, the correlations can be defined in an overall sense, that is, without respect to a given partition. ${ }^{26,64,73}$ The $k$-partitionability correlation and the $k$-producibility correlation $\operatorname{are}^{26}$

$$
\begin{aligned}
C_{k \text {-part }}(L) & :=\min _{\xi:|\xi| \geq k} C(\xi), \\
C_{k \text {-prod }}(L) & :=\min _{\xi: \forall X \in \xi,|X| \leq k} C(\xi)
\end{aligned}
$$

for $1 \leq k \leq|L|$. These characterize the strength of two different (one-parameter) notions of multiorbital correlations, those which cannot be restricted inside at least $k$ parts and those which cannot be restricted inside parts of size at most $k$, respectively. ${ }^{26}$

For the cluster $L$, as special cases, $C_{|L| \text {-part }}=C_{1 \text {-prod }}=C(\perp)$ grabs all the correlations; it is zero if and only if there is no correlation at all in the cluster $L$. On the other hand, $C_{2 \text {-part }}=C_{(|L|-1) \text {-prod }}$ is sensitive only for the strongest correlations, and it is nonzero if and only if the cluster $L$ is globally correlated. Note also that $C_{1 \text {-part }}=C_{|L| \text {-prod }}=C(T)=0$, by definition. Beyond these, there are no such coincidences among the partitionability and producibility correlations for other values of $k$; however, the relation $C_{k \text {-part }}$ $\geq C_{(|L|-k+1) \text {-prod }}$ holds. ${ }^{26}$ Also, the following (non strict) bounds hold: ${ }^{2}$

$$
\begin{gathered}
C_{k \text {-part }} \leq 2(k-1), \\
C_{k \text {-prod }} \leq 2(|L|-k) .
\end{gathered}
$$

\section{COMPUTATIONAL DETAILS}

In case of diborane molecules, the ground state geometries were optimized with the B3LYP/cc-pVDZ method. For the multiorbital correlation studies, the Pipek-Mezey ${ }^{88}$ localized HF/STO-3G molecular orbitals (MOs) were employed ${ }^{20,26}$ and they were manually hybridized (rotated) to better reflect the chemical environment. The application of the chemically intuitive STO-3G basis is supported by former findings on the robustness of chemical bonding measures. ${ }^{89-91}$ The adequacy of the standard minimal basis set is also corroborated in our previous work, i.e., comparing results obtained both in the standard STO-3G basis set (see the supplementary of Ref. 26) and molecule specific optimized minimal basis set $^{92-94}$ (see the main text of Ref. 26), we found negligible differences.

The quantum chemical (QC-)DMRG method was applied to study the multiorbital correlations in the full orbital following the procedure outlined in Ref. 26. The reduced density matrix was calculated by a method using the concept of generalized correlation functions, ${ }^{79}$ i.e., based on the expectation values of adequate 
n-orbital transition operators. The method was implemented in the QC-DMRG code by a method using the concept of generalized correlation functions.

The ground state geometries of both forms of the beryllium complex, namely, $\mathrm{Be}(\mathrm{CAC})_{2}$ and $\left[\mathrm{Be}(\mathrm{CAC})_{2}\right]^{2+}$, were taken from Ref. 41, and they correspond to the BP86/def2-TZVPP level of theory. In case of the beryllium complex, we have chosen a different strategy than the one employed for diboranes. Since we were interested only in the bonding of the $\mathrm{C}-\mathrm{Be}-\mathrm{C}$ atomic core, we have selected the complete active space (CAS) of relevant orbitals participating or influencing these bonds. In particular, $2 \mathrm{p}_{\mathrm{x}}$ orbitals on both $\mathrm{C}$ and $\mathrm{Be}$ atoms and the $2 \mathrm{~s}$ orbital on Be contribute to the $\sigma$ bonds and $2 \mathrm{p}_{z}$ orbitals on both $\mathrm{C}$ and neighboring $\mathrm{N}$ and Be atoms, forming or directly influencing the $\pi$ bonds. The remaining bonds were excluded since independent covalent bonds are uncorrelated in our analysis. ${ }^{26}$ For a numerical verification, see Fig. 9 in Appendix B. The CAS orbitals were optimized by means of the CASSCF $(10,9) / \mathrm{cc}-\mathrm{pVDZ}$ method in case of the neutral complex and the CASSCF $(8,9) / c c-p V D Z$ method in case of the dication and again localized using the Pipek-Mezey ${ }^{88}$ procedure. They were not hybridized in order to directly compare with the previous work ${ }^{41}$ making conclusions about atomiclike orbitals.

All the quantum chemistry calculations except the QC-DMRG ones were performed with the MOLPRO package. ${ }^{95}$ The QC-DMRG calculations were carried out using the Budapest QC-DMRG code. ${ }^{96}$ Molecular orbitals were visualized with Charmol. ${ }^{97}$ The numerical accuracy of the QC-DMRG calculations was controlled by the dynamic block state selection approach ${ }^{2,17,98}$ keeping up to thousands of block states for the a priori set quantum information loss threshold value $\chi=10^{-6}$. Initialization, i.e., optimal ordering of orbitals and dynamical extension of the active space, vital for quantum chemical DMRG calculations, was set up as discussed in Ref. 17.

\section{RESULTS}

The results on diborane(6) are summarized in Fig. 3 and Table I, whereas the results on diborane(4) are presented in Fig. 4 and Table II. All the figures depict mutual information of pairs of localized orbitals, defined in Eq. (2), while the tables contain numeric values of measures of the relevant kinds of multiorbital correlations, which are discussed below. In a similar fashion, the results on beryllium complexes are presented in Figs. 5, 7, and 8 and Table III.

In the figures, individual localized orbitals are represented as black dots and dashed blue lines encircle orbitals belonging to one atom. The mutual information is plotted as grayscaled edges between the orbitals. Black lines correspond to the strongest correlations, while light gray lines connect the weakly correlated orbitals. Based on the mutual information structure, the orbitals are grouped into strongly correlated clusters, which in our examples correspond to either core orbitals or chemical bonds. The clusters are encircled by red borders.

As the actual values of the dominant correlations are hard to read from the grayscaled graphs, for better visibility, they are also presented in the insets of the graphs in Fig. 10 in Appendix B.

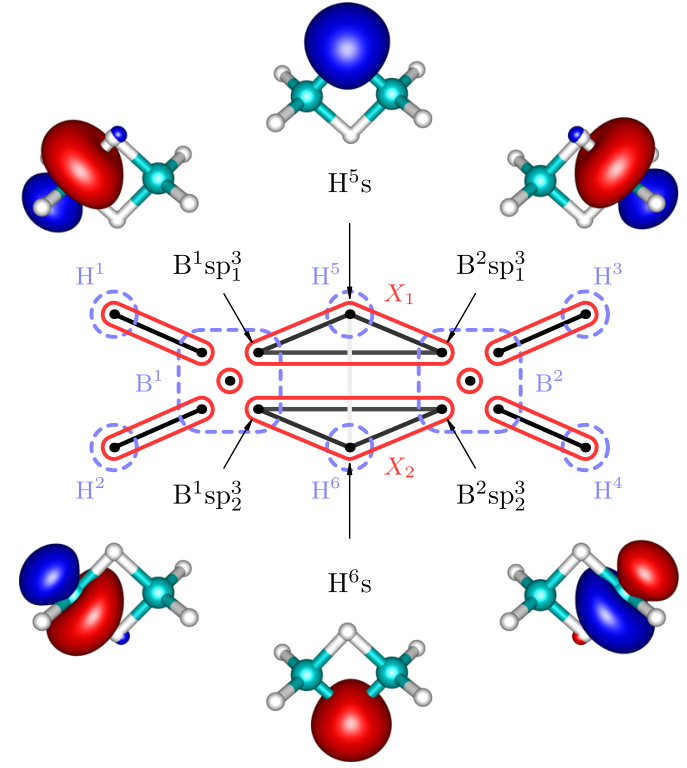

FIG. 3. Schematic view of diborane(6) with mutual information: each dot represents a localized orbital, dashed blue lines encircle individual atoms, edges correspond to mutual information (the plot shaded by a logarithmic scale depending on strength), and red circles show how the orbitals group into clusters, i.e., independent bonds. Sorted values of mutual information are plotted in Fig. 10 in Appendix B.

\section{DISCUSSION}

\section{Diborane(6)}

Figure 3 shows that our results are in good agreement with the established bonding picture of diborane(6) with two bridging $\mathrm{B}-\mathrm{H}-\mathrm{B}$ bonds. Let us now discuss in detail how the analysis of correlations leads to the clustering and to the bonding picture presented in Fig. 3. We will discuss only the bridging bonds; the core orbitals as well as terminal $\mathrm{B}-\mathrm{H}$ bonds are well separated, i.e., not

TABLE I. Correlation measures for diborane(6). Relative values are related to the upper bounds. Labeling of localized orbitals corresponds to Fig. 3 .

\begin{tabular}{lcc}
\hline \hline Correlation & Abs. value & Rel. value (\%) \\
\hline$C\left(X_{1} \mid\right.$ rest $)$ & 0.515 & 8.6 \\
$C\left(X_{1} \cup X_{2} \mid\right.$ rest $)$ & 0.412 & 3.4 \\
$C\left(\mathrm{~B}^{1} \mathrm{sp}_{1}^{3}, \mathrm{H}^{5} \mathrm{~s} \mid\right.$ rest $)$ & 1.852 & 46 \\
$C\left(\mathrm{H}^{5} \mathrm{~s}, \mathrm{~B}^{2} \mathrm{sp}_{1}^{3} \mid\right.$ rest $)$ & 1.852 & 46 \\
$C\left(\mathrm{~B}^{1} \mathrm{sp}_{2}^{3}, \mathrm{~B}^{2} \mathrm{sp}_{1}^{3} \mid\right.$ rest $)$ & 2.114 & 53 \\
$C\left(\mathrm{~B}^{1} \mathrm{sp}_{1}^{3} \mid \mathrm{H}^{5} \mathrm{~s}\right)$ & 0.894 & 45 \\
$C\left(\mathrm{H}^{5} \mathrm{~s} \mid \mathrm{B}^{2} \mathrm{sp}_{1}^{3}\right)$ & 0.894 & 45 \\
$C\left(\mathrm{~B}^{1} \mathrm{sp}_{1}^{3} \mid \mathrm{B}^{2} \mathrm{sp}_{1}^{3}\right)$ & 0.605 & 30 \\
$C_{2 \text {-part }}\left(X_{1}\right)$ & 1.500 & 75 \\
$C_{3-\text { part }}\left(X_{1}\right)$ & 2.394 & 60 \\
$C\left(X_{1} \mid X_{2}\right)$ & 0.309 & 5.2 \\
$C\left(\mathrm{H}^{5} \mathrm{~s} \mid \mathrm{H}^{6} \mathrm{~s}\right)$ & 0.042 & 2.1 \\
\hline \hline
\end{tabular}




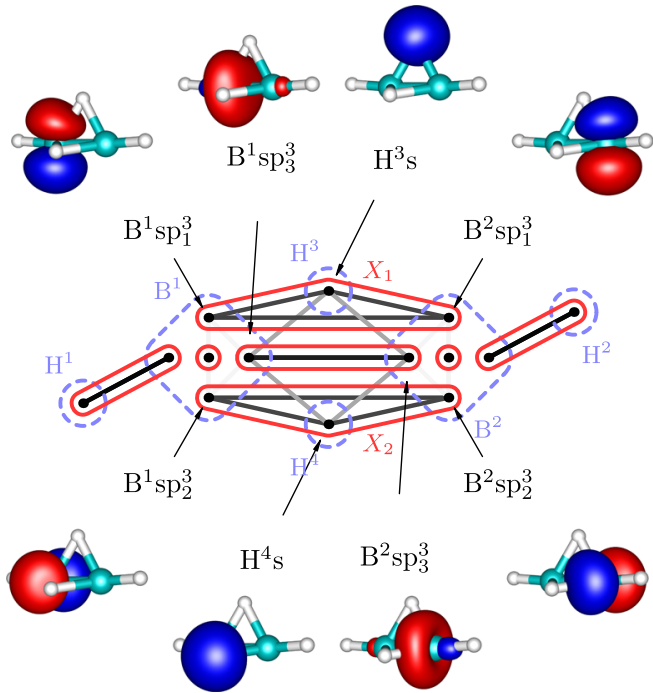

FIG. 4. Schematic view of diborane(4) with mutual information: each dot represents a localized orbital, dashed blue lines encircle individual atoms, edges correspond to mutual information (the plot shaded by a logarithmic scale depending on strength), and red circles show how the orbitals group into clusters, i.e., independent bonds. Sorted values of mutual information are plotted in Fig. 10 in Appendix B.

correlated with the rest. (This is confirmed by the weak correlation $C\left(X_{1} \cup X_{2} \mid\right.$ rest $)$ in Table I. $)$

First, we consider the cluster $X_{1}$ containing $\mathrm{sp}^{3}$ hybrid orbitals on $\mathrm{B}$ atoms and the $1 \mathrm{~s}$ orbital on the bridging $\mathrm{H}$ atom. Because of the point group symmetry, the same results hold for cluster $X_{2}$. As can be seen in Table I, the correlation (entanglement) of $X_{1}$ with the remaining orbitals is very weak, only $8.6 \%$ of the maximum value, which indicates that $X_{1}$ forms an independent three-center bond.
TABLE II. Correlation measures for diborane(4). Relative values are related to the upper bounds. Labeling of localized orbitals corresponds to Fig. 4.

\begin{tabular}{lcc}
\hline \hline Correlation & Abs. value & Rel. value (\%) \\
\hline$C\left(X_{1} \mid\right.$ rest $)$ & 1.328 & 22 \\
$C\left(\mathrm{~B}^{1} \mathrm{sp}_{1}^{3} \mid \mathrm{H}^{3} \mathrm{~s}\right)$ & 0.647 & 32 \\
$C\left(\mathrm{~B}^{1} \mathrm{sp}_{1}^{3} \mid \mathrm{B}^{2} \mathrm{sp}_{1}^{3}\right)$ & 0.701 & 35 \\
$C_{2 \text {-part }}\left(X_{1}\right)$ & 1.388 & 69 \\
$C_{3 \text {-part }}\left(X_{1}\right)$ & 2.089 & 52 \\
$C\left(X_{3} \mid\right.$ rest $)$ & 1.438 & 36 \\
$C\left(\mathrm{~B}^{1} \mathrm{sp}_{3}^{3} \mid \mathrm{B}^{2} \mathrm{sp}_{3}^{3}\right)$ & 1.245 & 62 \\
$C\left(\mathrm{~B}^{1} \mathrm{sp}_{3}^{3} \mid \mathrm{H}^{3} \mathrm{~s}\right)$ & 0.130 & 6.5 \\
$C\left(X_{1} \cup X_{2} \cup X_{3} \mid\right.$ rest $)$ & 0.535 & 6.7 \\
$C\left(X_{1} \mid X_{2}\right)$ & 0.066 & 1.1 \\
$C\left(X_{1} \mid X_{3}\right)$ & 0.639 & 16 \\
\hline
\end{tabular}

However, to confirm this conclusion, we have to show that $X_{1}$ cannot be split further. If we take separate pairs of orbitals from $X_{1}$ and measure the correlation with the remaining orbitals, we obtain significantly higher values, in particular, $46 \%$ and $53 \%$ of the maximum values (see Table I), which justifies existence of the three-center bond.

The mutual information of pairs of orbitals within $X_{1}$ reaches rather small relative values ( $45 \%$ and $30 \%$; see Table I); however, according to our numerical experience, even strong multicenter bonds typically yield low percentage, never approaching near the theoretical maxima. ${ }^{26}$ Intuitive perspective suggests that the correlation of one orbital with the others can be thought of as a resource shared among the orbitals. In other words, all the pairs inside $X_{1}$ cannot reach the maximum simultaneously, bounded by entanglement monogamy. ${ }^{99,100}$ The formulation of an inequality bounding the mutual correlations inside orbital clusters still remains an open problem to our best knowledge. The smaller value of the mutual

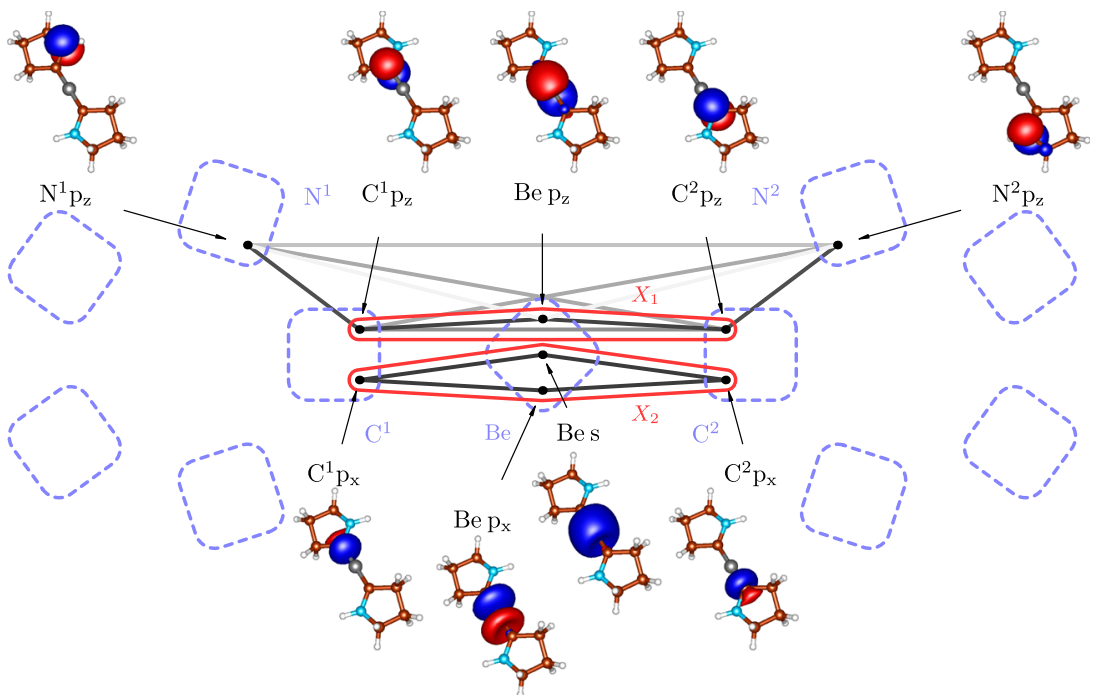

FIG. 5. Schematic view of $\mathrm{Be}(\mathrm{CAC})_{2}$ with mutual information. In order to be consistent with Figs. 3 and 4, all the atoms are depicted (dashed blue lines), even though only a subset of orbitals (black dots) formed the complete active space. Note that one ring is artificially flipped for clearer correlation picture. Sorted values of mutual information are plotted in Fig. 10 in Appendix B. 
TABLE III. Correlation measures for $\mathrm{Be}(\mathrm{CAC})_{2}$. Relative values are related to the upper bounds. Labeling of localized orbitals corresponds to Fig. 5.

\begin{tabular}{|c|c|c|}
\hline Correlation & Abs. value & Rel. value (\%) \\
\hline$C\left(C^{1} p_{z}, C^{2} p_{z} \mid\right.$ rest $)$ & 3.424 & 86 \\
\hline$C\left(\mathrm{C}^{1} \mathrm{p}_{\mathrm{z}}, \mathrm{Be} \mathrm{p}_{\mathrm{z}} \mid\right.$ rest $)$ & 2.432 & 61 \\
\hline$C\left(X_{1} \mid\right.$ rest $)$ & 2.032 & 34 \\
\hline$C\left(C^{1} p_{z} \mid C^{2} p_{z}\right)$ & 0.194 & 9.7 \\
\hline$C\left(\mathrm{C}^{1} \mathrm{p}_{\mathrm{z}} \mid \mathrm{Be} \mathrm{p}_{\mathrm{z}}\right)$ & 0.681 & 34 \\
\hline$C_{2-\text { part }}\left(X_{1}\right)$ & 1.153 & 58 \\
\hline$C_{3-\text { part }}\left(X_{1}\right)$ & 1.834 & 46 \\
\hline$C\left(X_{1} \mid \mathrm{N}^{1} \mathrm{p}_{z}\right)$ & 0.915 & 46 \\
\hline$C\left(X_{1}^{\prime} \mid\right.$ rest $)$ & 0.209 & 2.6 \\
\hline$C\left(\mathrm{~N}^{1} \mathrm{p}_{\mathrm{z}}, \mathrm{C}^{1} \mathrm{p}_{\mathrm{z}} \mid\right.$ rest of $\left.X_{1}^{\prime}\right)$ & 1.737 & 43 \\
\hline$C\left(\mathrm{~N}^{1} \mathrm{p}_{\mathrm{z}} \mid \mathrm{C}^{1} \mathrm{p}_{\mathrm{z}}\right)$ & 0.560 & 28 \\
\hline$C\left(X_{2} \mid\right.$ rest $)$ & 0.209 & 2.6 \\
\hline$C\left(\mathrm{C}^{1} \mathrm{p}_{\mathrm{x}} \mid \mathrm{Be} \mathrm{s}\right)$ & 0.765 & 38 \\
\hline$C\left(C^{1} p_{x} \mid B e p_{x}\right)$ & 0.771 & 39 \\
\hline$C_{2 \text {-part }}\left(X_{2}\right)$ & 1.936 & 97 \\
\hline$C\left(\mathrm{C}^{1} \mathrm{p}_{\mathrm{x}} \mid \mathrm{Be} \mathrm{sp}_{2}\right)$ & 1.912 & 96 \\
\hline
\end{tabular}

information between $\mathrm{sp}^{3}$ hybrid orbitals on $\mathrm{B}$ atoms reflects their larger internuclear distance.

We employ $k$-partitionability in order to quantify and benchmark the strength of the diborane(6) three-center bonds (in terms of correlation). As can be seen in Table I, $C_{2 \text {-part }}\left(X_{1}\right)$ reaches $75 \%$ of the upper bound and $C_{3 \text {-part }}\left(X_{1}\right) 60 \%$, which points at a strong bond in $X_{1}$.

The very weak correlation (entanglement) of $X_{1}$ with the remaining orbitals also indicates that the state of the cluster $X_{1}$ is close to a pure state. Indeed, the eigenstate analysis of the reduced density operator $\rho_{X_{1}}$ shows that there is the following twoelectron eigenstate with a corresponding eigenvalue (probability) of 0.94:

$$
\begin{aligned}
\left|\psi_{X_{1}}\right\rangle= & +0.2146|--\uparrow \downarrow\rangle+0.4313|-\uparrow \downarrow-\rangle+0.2146|\uparrow \downarrow--\rangle \\
& +0.3787|-\downarrow \uparrow\rangle-0.3787|-\uparrow \downarrow\rangle+0.2721|\downarrow-\uparrow\rangle \\
& +0.3787|\uparrow \downarrow-\rangle-0.3787|\downarrow \uparrow-\rangle-0.2721|\uparrow-\downarrow\rangle,
\end{aligned}
$$

where the ordering of orbitals in a ket corresponds to $B^{1} \mathrm{sp}^{3}, \mathrm{H}^{5} 1 \mathrm{~s}$, and $\mathrm{B}^{2} \mathrm{sp}^{3}$. Other eigenstates have probabilities below 0.01 . The principal two-electron eigenstate together with the above discussion on correlations implies that the three orbitals of $X_{1}$ form a three-center two-electron bond. Note that the electron pair exhibits a preferred occupation on the $\mathrm{H}$ atom, which is due to its higher electronegativity when compared to $\mathrm{B}$, as we can see from the principal eigenstate. It is in agreement with the expectation values of particle-numberoperators $\left(\left\langle\psi\left|\hat{n}_{\mathrm{el}}^{(i)}\right| \psi\right\rangle\right)$ which for $\mathrm{B}^{1} \mathrm{sp}^{3}, \mathrm{H}^{5} 1 \mathrm{~s}$, and $\mathrm{B}^{2} \mathrm{sp}^{3}$ equal 0.53 , 0,95 , and 0.53 , respectively.

As one can observe in Table I, the main source of correlation between $X_{1}$ and the remaining orbitals is the correlation with the other three-center bond, $X_{2}$. Specifically, the correlation between two bridging $\mathrm{H}$ atoms is the strongest, which is caused by higher electron density on these atoms.

\section{Diborane(4)}

In case of diborane(4), the two terminal $\mathrm{H}$ atoms are missing and instead a direct covalent bond connecting both $\mathrm{B}$ atoms is present. ${ }^{40}$ This is also the picture resulting from our analysis and depicted in Fig. 4. In comparison to diborane(6), we have the similar three-orbital clusters $X_{1}$ and $X_{2}$ but also the two-orbital cluster $X_{3}$ containing $\mathrm{sp}^{3}$ hybrid orbitals on $\mathrm{B}$ atoms and corresponding to the aforementioned B-B bond.

Considering the cluster $X_{1}$, one can observe in Table II that it is more correlated with the remaining orbitals than in the case of diborane(6). The value of $C\left(X_{1} \mid\right.$ rest $)$ is more than two times larger, but the picture of $X_{1}$ as a standalone chemical bond is still justifiable. Consequently, the reduced density operator $\rho_{X_{1}}$ is more mixed, with the principal eigenvalue of 0.7803 . The remaining eigenstates share low probabilities (below 0.081), and therefore, the picture of $X_{1}$ as a standalone chemical bond is still a reasonable qualitative description. The principal eigenstate is again two-electron, i.e., electron-deficient, and it has the following form:

$$
\begin{aligned}
\left|\psi_{X_{1}}\right\rangle= & -0.2287|--\uparrow \downarrow\rangle-0.3671|-\uparrow \downarrow-\rangle-0.2287|\uparrow \downarrow--\rangle \\
& +0.3823|-\downarrow \uparrow\rangle-0.3823|-\uparrow \downarrow\rangle-0.2967|\downarrow-\uparrow\rangle \\
& +0.3823|\uparrow \downarrow-\rangle-0.3823|\downarrow \uparrow-\rangle+0.2967|\uparrow-\downarrow\rangle .
\end{aligned}
$$

Similarly to diborane(6), higher electron density is on the bridging $\mathrm{H}$ atom, which is due to its higher electronegativity.

Comparing the two-orbital correlations inside $X_{1}$ with diborane(6) (Tables I and II), one can see a weaker correlation between $\mathrm{sp}^{3}$ hybrid orbitals on $\mathrm{B}$ atoms and $\mathrm{H}$ 1s orbital but a slightly stronger correlation between both $\mathrm{B}$-atom-orbitals. This stronger correlation can be certainly assigned to a shorter distance of B atoms (1.477 $\AA$ vs $1.784 \AA$ ). Based on the values of $C_{2 \text {-part }}\left(X_{1}\right)$ and $C_{3 \text {-part }}\left(X_{1}\right)$, the covalent bond corresponding to the cluster $X_{1}$ is slightly weaker (in terms of correlation) than the same bond in diborane(6).

For the cluster $X_{3}=\left\{\mathrm{B}^{1} \mathrm{sp}_{3}^{3}, \mathrm{~B}^{2} \mathrm{sp}_{3}^{3}\right\}$, correlation with the remaining orbitals is stronger than for $X_{1}$ and $X_{2}$, which in turn weakens the internal two-orbital correlation. The major contribution to the correlation of $X_{3}$ with the remaining orbitals originates from $C\left(\mathrm{~B}^{1} \mathrm{sp}_{3}^{3} \mid \mathrm{H}^{3} \mathrm{~s}\right)$, which is still very weak compared to other correlations in the molecule (see Table II).

The overall correlation of the three bonding clusters $X_{1}, X_{2}$, and $X_{3}$ with the rest of the system is similarly weak as in diborane(6), so the considered bonding can be described independently of the rest of the molecule.

\section{Beryllium complexes}

In order to check how well our bonding picture of $\mathrm{Be}(\mathrm{CAC})_{2}$ fits the one proposed by Arrowsmith et al., ${ }^{41}$ we consider the clusters $X_{1}$ and $X_{2}$ from Fig. 5. The cluster $X_{1}$ contains $\mathrm{p}_{z}$ orbitals on $\mathrm{C}$ and $\mathrm{Be}$ atoms and corresponds to the suggested three-center twoelectron $\pi$ bond, whereas the cluster $X_{2}$ contains $C p_{x}$ orbitals and Be $s$ and $\mathrm{p}_{\mathrm{x}}$ orbitals and corresponds to the $\sigma$ bonds.

Let us start with $X_{1}$. In Table III, one can see that the correlation of $X_{1}$ with the remaining orbitals is higher than $30 \%$ of the maximum value, which means that the picture of the three-orbital $\mathrm{C}-\mathrm{Be}-\mathrm{C} \pi$ bond might be good as a qualitative description, but for a quantitatively adequate description, we might seek to include further orbitals into $\mathrm{X}_{1}$, as shown below. This is also demonstrated by 


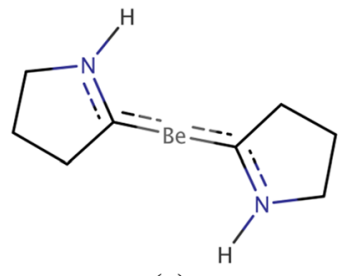

(a)

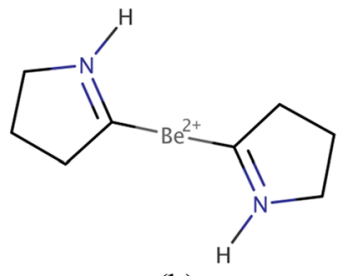

(b)
FIG. 6. Structures of studied beryllium complexes (a) $\mathrm{Be}(\mathrm{CAC})_{2}$ and (b) $\left[\mathrm{Be}(\mathrm{CAC})_{2}\right]^{2+}$ suggested by our correlation analysis.

weaker pairwise correlations within $X_{1}$, especially $C\left(C^{1} \mathrm{p}_{\mathrm{z}} \mid \mathrm{C}^{2} \mathrm{p}_{\mathrm{z}}\right)$, than in the three-orbital bonds discussed above. The correlations of the internal pairs in $X_{1}$ with the remaining orbitals are large $(61 \%$ and $86 \%$ ) and clearly cannot be considered as standalone bonds.

The inaccuracy of the picture of a standalone three-orbital bond is also demonstrated by the reduced density operator $\rho_{X_{1}}$ (see in Appendix A), which is much more mixed than in previous cases. It has three dominant eigenvalues instead of just one. The most significant, nevertheless, corresponds to the two-electron state, which is in agreement with the overall picture of the three-orbital two-electron bond.

As can be seen in Fig. 5, the strongest external correlation of $X_{1}$ is $C\left(X_{1} \mid \mathrm{N}^{1} \mathrm{p}_{\mathrm{z}}\right)$. It results from a conjugation of $\mathrm{p}_{z}$ orbitals and has a stabilization effect. Notice that the $\mathrm{N}^{1}-\mathrm{C}^{1}-\mathrm{Be}-\mathrm{C}^{2}-\mathrm{N}^{2}$ group of atoms forms perfectly planar structure (the dihedral angle $\alpha_{\mathrm{N}-\mathrm{C}-\mathrm{C}-\mathrm{N}}$ $=179.97^{\circ}$ ) enabling an efficient overlap of all $\mathrm{p}_{z}$ orbitals, which is necessary for the aforementioned conjugation.

The more accurate bonding picture can thus be obtained by considering the enlarged cluster $X_{1}^{\prime}$

$$
X_{1} \longmapsto X_{1}^{\prime} \equiv X_{1} \cup\left\{\mathrm{N}^{1} \mathrm{p}_{\mathrm{z}}, \mathrm{N}^{2} \mathrm{p}_{\mathrm{z}}\right\} .
$$

The corresponding structure of $\mathrm{Be}(\mathrm{CAC})_{2}$ is depicted in Fig. 6(a). The cluster $X_{1}^{\prime}$ is independent of the rest of the molecule. This follows from the negligible correlation of $X_{1}^{\prime}$ with the remaining orbitals (see Table III). Employing the standard notation, the $\pi$ electron bond can be denoted as $\Pi_{5}^{6}$, i.e., the six-electron (two electrons from the Be atom and two from each $\mathrm{N}$ atom lone pair) five-center bond, which is confirmed by the particle number expectation value of 6.003 .

In order to verify the suggested $\pi$ back-donation mechanism ${ }^{41}$ or in other words to probe the local electronic configuration of the $\mathrm{Be}$ atom, we have also performed the correlation analysis for the dication $\left[\mathrm{Be}(\mathrm{CAC})_{2}\right]^{2+}$. As can be seen in Fig. 7, the difference between the correlation picture of $\mathrm{Be}(\mathrm{CAC})_{2}$ and $\left[\mathrm{Be}(\mathrm{CAC})_{2}\right]^{2+}$ is almost missing correlations inside the cluster $X_{1}$, which is, for example, demonstrated by the negligible value of $C_{2-\text { part }}\left(X_{1}\right)=0.093$. Also the reduced density operator $\rho_{X_{1}}$ is highly mixed and without the dominating two-electron eigenstate (see in Appendix A). On the other hand, the correlations in the cluster $X_{2}$ remain practically unchanged.

This is in agreement with the picture of the Be atom having originally two electrons in the $\mathrm{p}_{z}$ orbital. When the $\mathrm{C}-\mathrm{Be}-\mathrm{C}$ bond is formed, they are shared with $\mathrm{C}$-atom $\mathrm{p}_{z}$ orbitals through the back donation mechanism, as is depicted in Fig. 2. These two $\pi$ electrons are missing in case of the dication, and the aforementioned $\pi$ bond is clearly not formed.

Another feature of the correlation picture from Fig. 7 is that there are considerably stronger pairwise correlations between $\mathrm{C}$ and $\mathrm{N}$-atom $\mathrm{p}_{z}$ orbitals $\left[C\left(\mathrm{~N}^{1} \mathrm{p}_{\mathrm{z}} \mid \mathrm{C}^{1} \mathrm{p}_{\mathrm{z}}\right)=1.691\right]$. They are indeed the strength of donor-acceptor bonds. ${ }^{20}$ We thus assign double bonds between $\mathrm{N}$ and $\mathrm{C}$ atoms to the dication, as is depicted in Fig. 6(b). The $\pi$ bonds are formed from the originally doubly filled $\mathrm{N}_{z}$ orbitals and empty $\mathrm{C} \mathrm{p}_{z}$ orbitals. The existence of these $\pi$ bonds is also confirmed by the almost perfectly planar environment with the dihedral angle $\alpha_{\mathrm{H}-\mathrm{N}-\mathrm{C}-\mathrm{Be}}=1.26^{\circ}$. Note that in Fig. 7, we can see only the part of the double bond corresponding to the $\pi$ bond-the $\sigma$ bonds along the rings are excluded from the active space.

Let us now turn to the $X_{2}$ cluster of $\mathrm{Be}(\mathrm{CAC})_{2}$, i.e., to the $\sigma$ bonding. The correlation of $X_{2}$ with the remaining orbitals is

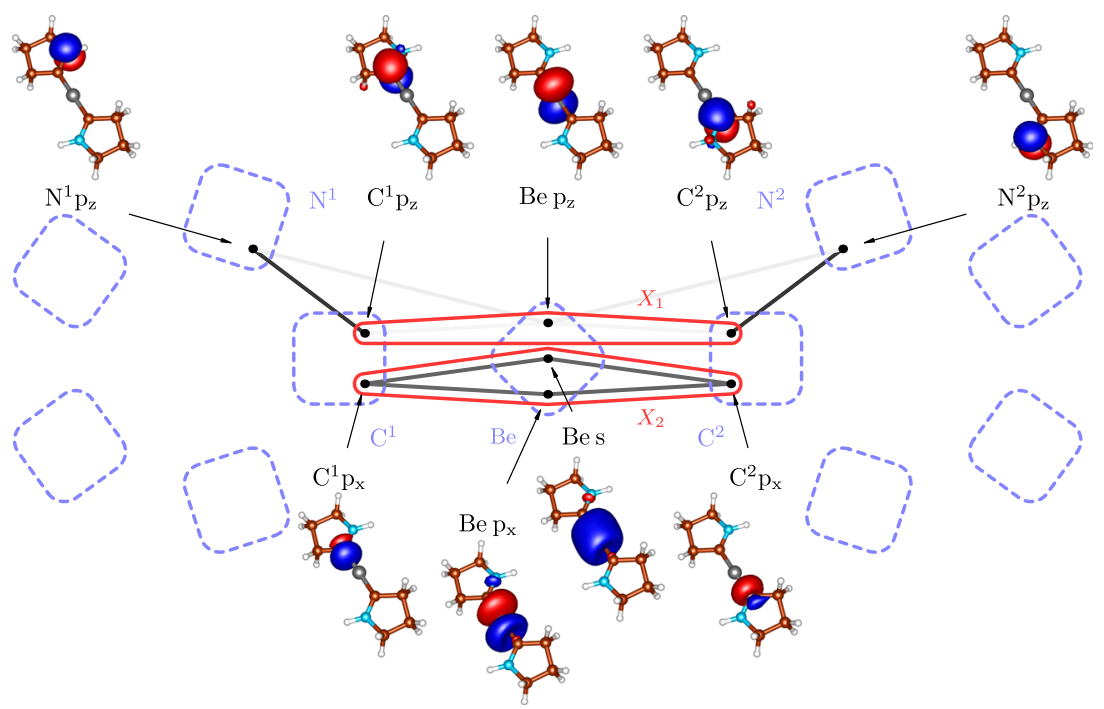

FIG. 7. Schematic view of $\left[\mathrm{Be}(\mathrm{CAC})_{2}\right]^{2+}$ with mutual information. Note that one ring is artificially flipped for clearer correlation picture. 


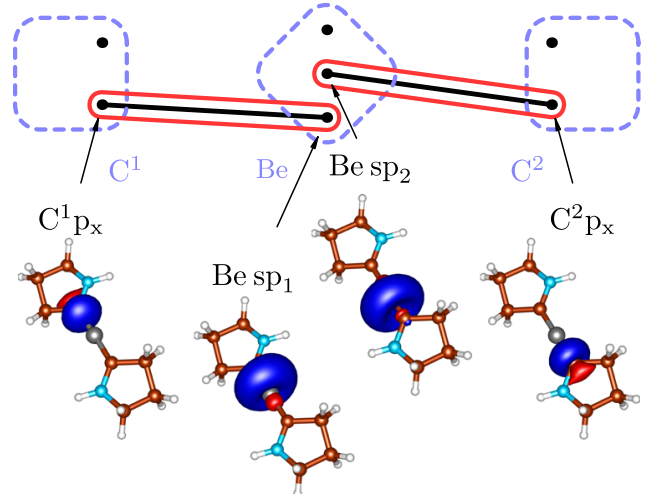

FIG. 8. Mutual information of the $\mathrm{Be}(\mathrm{CAC})_{2} \sigma$ bonding channel after the rotation of Be $s$ and Be $p_{x}$ orbitals.

insignificant; however, splitting of the four-orbital cluster into two $\sigma$ bonds is not possible in this basis. It may therefore seem that the correct $\sigma$ bond is also multiorbital. It is, however, merely the artifact of the atomiclike basis, which was used in order to directly compare with Ref. 41 . By simple rotation of Be $s$ and $\mathrm{p}_{x}$ orbitals (forming the sp hybrids as in the case of diboranes), one can form two independent $\sigma$ bonds, essentially without influencing the rest of the system, which can be seen in Fig. 8. Note that in the correlation theory of the chemical bond, superposing orbitals is allowed if this does not affect their locality too much. So superposing orbitals on different atoms is usually forbidden, while doing the same on a given atom is allowed.

Last but not least, we would like to compare the strength of both contributions to the bonding of the $\mathrm{C}-\mathrm{Be}-\mathrm{C}$ core, namely, the $\pi$ bond (clusters $X_{1}$ and $X_{1}^{\prime}$ ) and the $\sigma$ bond (cluster $X_{2}$ ). In the previous study, ${ }^{41}$ it was shown by means of the energy decomposition analysis combined with natural orbitals for chemical valence (EDANOCV $)^{101}$ that the $\pi$ bonding channel is considerably more energy stabilizing than the $\sigma$ channel. Let us now check the strength of both contributions by means of the correlations.

When using the more rough (three-orbital two-electron) description of the $\pi$ bond (cluster $X_{1}$ ) and by looking at Table III, one can see on the values of $C_{2-\text { part }}$ that $\sigma$ bonds are considerably stronger than the $\pi$ bond. The situation, however, dramatically changes when we use more accurate conjugated description (cluster $\left.X_{1}^{\prime}\right)$. Note that since we are not interested in a split dissecting $\mathrm{N}$ and $\mathrm{C}$ (because the $\mathrm{N}-\mathrm{C}$ bond on the ring is stabilized by another $\sigma$ bond, not visible in the plots), we have to consider the $\mathrm{p}_{z}$ orbitals together on $\mathrm{N}$ and $\mathrm{C}$ atoms of the same ring and calculate $C_{2-\text { part }}\left(\mathrm{N}^{1} \mathrm{p}_{\mathrm{z}}, \mathrm{C}^{1} \mathrm{p}_{\mathrm{z}}\right.$ Be $\left.\mathrm{p}_{\mathrm{z}} \mid \mathrm{N}^{2} \mathrm{p}_{\mathrm{z}}, \mathrm{C}^{2} \mathrm{p}_{\mathrm{z}}\right)$, which turns out to be $C\left(\mathrm{~N}^{1} \mathrm{p}_{\mathrm{z}}, \mathrm{C}^{1} \mathrm{p}_{\mathrm{z}} \mid\right.$ rest of $\left.X_{1}^{\prime}\right)$. Also note that in this paragraph, we compared the absolute values of the correlation measures because the clusters are of different sizes.

In Table III, one can see that the more accurate description of the $\pi$ bond $\left(X_{1}^{\prime}\right)$ makes it of a similar strength as the $\sigma$ bonds (1.737 vs 1.936). We believe that our results describe the nature of a $\mathrm{Be}(\mathrm{CAC})_{2}$ bonding reliably, especially because we have used the genuine multireference description unlike in Ref. 41, where the analysis was based on the density functional theory (BP86 functional). We would also like to note that we have studied a slightly different system than in Ref. 41. In our case, all substituents were replaced by hydrogen atoms. This, however, should not influence the electronic structure of the $\mathrm{C}-\mathrm{Be}-\mathrm{C}$ core. Also note that using the $\mathrm{s}$ and $\mathrm{p}_{\mathrm{x}}$ orbitals on beryllium in $X_{2}$ was only for the purpose of comparison with the previous study. ${ }^{41}$ For having a more physical picture, we should use the hybridized orbitals (Fig. 8), by which $X_{2}$ consists of two simple covalent bonds. Then, in order to characterize the strength of the bond, we would have to consider the Be $\mathrm{sp}_{1}$ and $\mathrm{sp}_{2}$ orbitals together and calculate $C_{2-\text { part }}\left(\mathrm{C}^{1} \mathrm{p}_{\mathrm{x}}\left|\mathrm{Be} \mathrm{sp}_{1}, \mathrm{Be} \mathrm{sp}_{2}\right| \mathrm{C}^{2} \mathrm{p}_{\mathrm{x}}\right)$ $=1.935$. Nevertheless, since this value is nearly identical to $C_{2-\text { part }}\left(X_{2}\right)$, the conclusion is the same.

\section{CONCLUSIONS}

In this article, we have reviewed the recently developed correlation theory of the chemical bond ${ }^{26}$ and applied it on molecules with multicenter electron-deficient bonds. We have demonstrated the usefulness of our methodology in characterizing molecular bonding properties by fingerprints of correlations among individual orbitals which form these types of bonds.

We have verified the computational procedure on a textbook molecule with electron-deficient bonds, namely, diborane(6), and further characterized bonding in diborane(4) and zero-valent complexes of beryllium with intricate bonding patterns. In all the cases, our results fit well with known bonding pictures or previous theoretical predictions. We have therefore proved capabilities of our new method to reliably describe bonding in complex molecular systems.

In case of the $\mathrm{Be}(\mathrm{CAC})_{2}$ molecule, we have also compared both contributions to the $\mathrm{C}-\mathrm{Be}-\mathrm{C}$ bonding $(\sigma$ and $\pi$ ), finding, in contrast to the previous study, ${ }^{41}$ the $\sigma$ and $\pi$ contributions of a similar strength, in the sense of correlational quantities. We believe that our result is reliable and attribute the discrepancy with the previous study to the single reference description employed in Ref. 41, which may not be accurate enough in this multireference case.

Finally, we would like to note that despite employing the DMRG method ${ }^{35,36}$ for calculations of subsystem reduced density matrices, the theory presented in this article is general and other correlated methods can in principle be employed as well, ${ }^{16}$ especially in cases of large molecules with the electronic structure dominated by the dynamical correlation for which the DMRG description may be unnecessary and computationally prohibitive.

\section{ACKNOWLEDGMENTS}

This work has been supported by the Czech Science Foundation (Grant Nos. 16-12052S, 18-24563S, and 18-18940Y), the Czech Ministry of Education, Youth and Sports (Project No. LTAUSA17033), and the Hungarian-Czech Joint Research Project No. MTA/16/05. G.B., S.S., and Ö.L. were supported by the National Research, Development and Innovation Fund of Hungary (NRDIFH) within the Researcher-initiated Research Program (Project No. NKFIHK120569) and the "Lendület" Program of the Hungarian Academy of Sciences (HAS). S.S. and Ö.L. were supported by the Quantum Technology National Excellence Program (Project No. 20171.2.1-NKP-2017-00001) of NRDIFH. G.B. and S.S. were also supported by the "Bolyai" Research Scholarship of HAS. Ö.L. also acknowledges financial support from the Alexander von Humboldt foundation. 


\section{APPENDIX A: EIGENVECTORS OF THE REDUCED DENSITY OPERATORS}

\section{1. $\operatorname{Be}(\mathrm{CAC})_{2}$}

The (reduced) density operator of the $X_{1}$ orbitals consists of the following eigenstates of the three highest eigenvalues (probabilities):

Probability 0.5798

$$
\begin{aligned}
\left|\psi_{X_{1}}^{1}\right\rangle= & +0.0864|--\uparrow \downarrow\rangle+0.3255|-\downarrow \uparrow\rangle-0.3255|-\uparrow \downarrow\rangle \\
& +0.3324|-\uparrow \downarrow-\rangle+0.4748|\downarrow-\uparrow\rangle-0.3254|\downarrow \uparrow-\rangle \\
& -0.4748|\uparrow-\downarrow\rangle+0.3254|\uparrow \downarrow-\rangle+0.0863|\uparrow \downarrow--\rangle,
\end{aligned}
$$

Probability 0.1473

$$
\begin{aligned}
\left|\psi_{X_{1}}^{2}\right\rangle= & -0.2010|-\downarrow \uparrow \downarrow\rangle-0.2630|-\uparrow \downarrow \downarrow\rangle-0.3980|\downarrow-\uparrow \downarrow\rangle \\
& -0.2781|\downarrow \downarrow \uparrow\rangle+0.5562|\downarrow \uparrow \downarrow\rangle-0.2630|\downarrow \uparrow \downarrow-\rangle \\
& -0.2781|\uparrow \downarrow \downarrow\rangle-0.3980|\uparrow \downarrow-\downarrow\rangle+0.2010|\uparrow \downarrow \downarrow-\rangle,
\end{aligned}
$$

Probability 0.1473

$$
\begin{aligned}
\left|\psi_{X_{1}}^{3}\right\rangle= & +0.2010|-\uparrow \uparrow \downarrow\rangle+0.2630|-\uparrow \downarrow \uparrow\rangle-0.2781|\downarrow \uparrow \uparrow\rangle \\
& +0.3980|\uparrow-\uparrow \downarrow\rangle+0.5562|\uparrow \downarrow \uparrow\rangle-0.2781|\uparrow \uparrow \downarrow\rangle \\
& +0.2630|\uparrow \uparrow \downarrow-\rangle+0.3980|\uparrow \downarrow-\uparrow\rangle-0.2010|\uparrow \downarrow \uparrow-\rangle ;
\end{aligned}
$$

all the other eigenvalues are less than 0.033 .

\section{2. $\left[\mathrm{Be}(\mathrm{CAC})_{2}\right]^{2+}$}

As mentioned earlier in the text, the reduced density operator for $X_{1}$ is highly mixed in this case, with no dominant state. Therefore, we only list the highest eigenvalues to show this

$$
0.1016,0.1007,0.1007,0.1007,0.1002 \text {, }
$$$$
0.1002,0.0899,0.0899,0.0868,0.0214 \text {. }
$$

\section{APPENDIX B: SUPPLEMENTARY CORRELATION PLOTS}

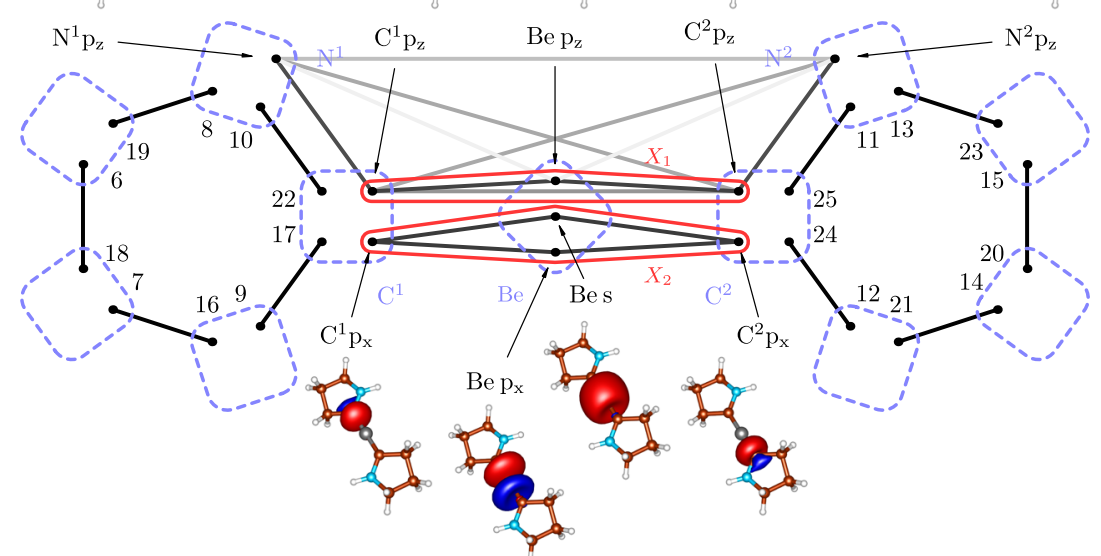

(a)
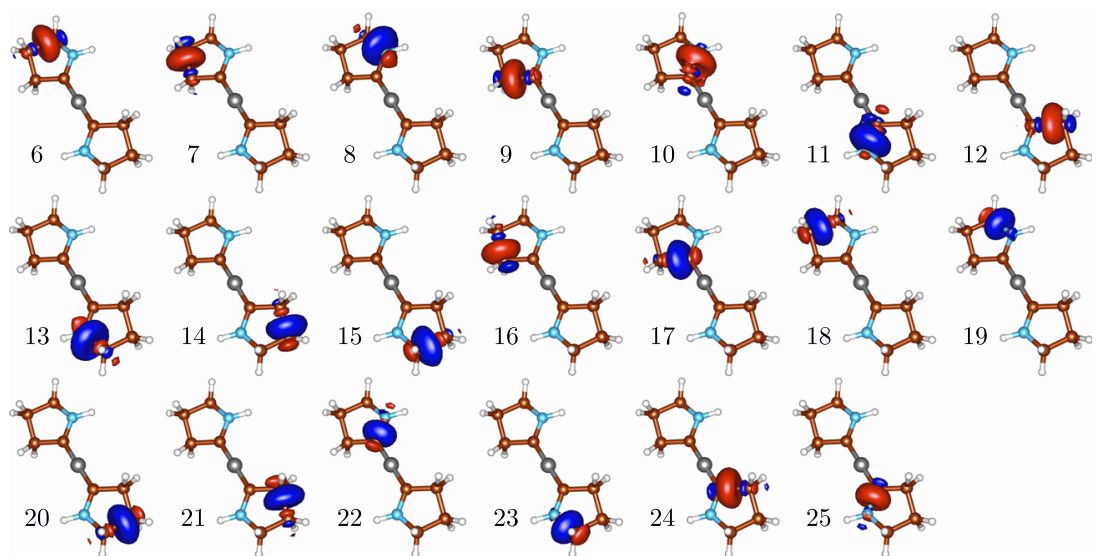

(b)
FIG. 9. (a) Schematic plot of mutual information in $\mathrm{Be}(\mathrm{CAC})_{2}$ with the active space extended to include the orbitals responsible for the sigma-bonding. (b) The numbering of orbitals corresponds to the individual orbital pictures. After extending the active space, correlations remain virtually unchanged, with differences well below $1 \%$. 


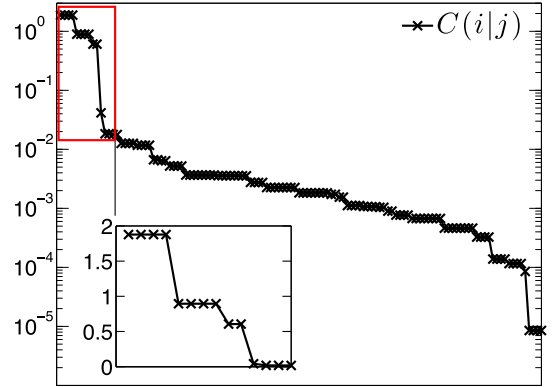

(a)

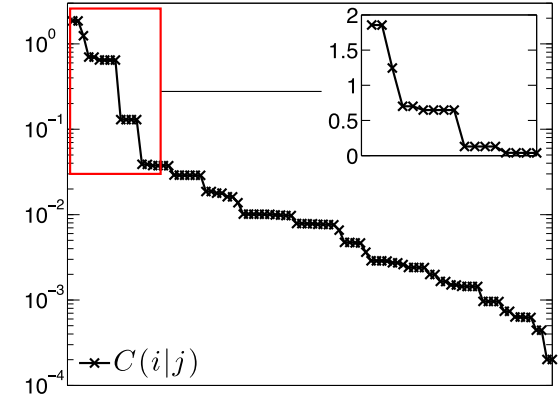

(b)

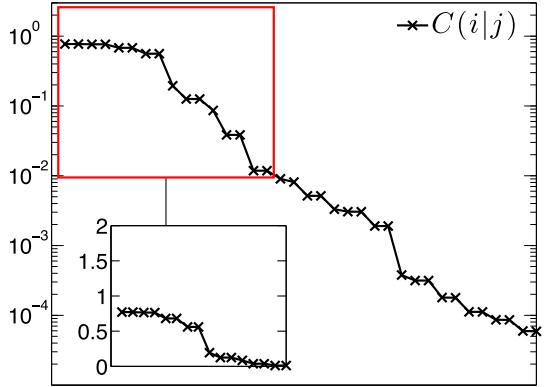

(c)

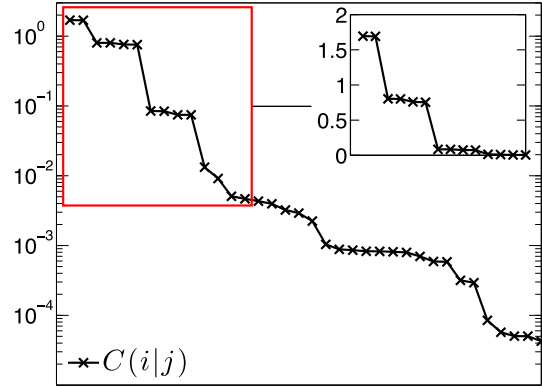

(d)

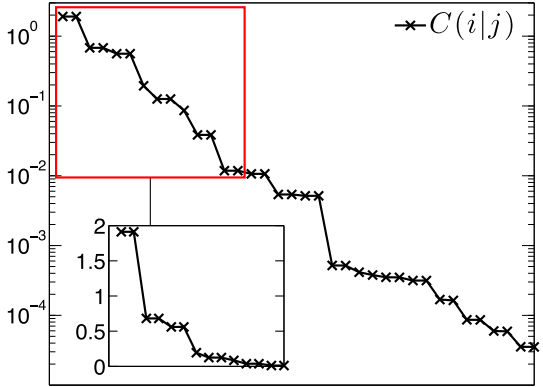

(e)

FIG. 10. The distributions of the two-orbital correlations for (a) diborane(6), (b) diborane(4), and beryllium complexes (c) $\mathrm{Be}(\mathrm{CAC})_{2},(\mathrm{~d})\left[\mathrm{Be}(\mathrm{CAC})_{2}\right]^{2+}$, and $(\mathrm{e}) \mathrm{Be}(\mathrm{CAC})_{2}$ with rotated $X_{2}$.

\section{REFERENCES}

º̈. Legeza and J. Sólyom, Phys. Rev. B 68, 195116 (2003).

${ }^{2}$ Ö. Legeza and J. Sólyom, Phys. Rev. B 70, 205118 (2004).

${ }^{3}$ Z. Huang and S. Kais, Chem. Phys. Lett. 413, 1 (2005).

${ }^{4}$ J. Rissler, R. M. Noack, and S. R. White, Chem. Phys. 323, 519 (2006).

${ }^{5}$ J. Pipek and I. Nagy, Phys. Rev. A 79, 052501 (2009).

${ }^{6}$ G. Barcza, Ö. Legeza, K. H. Marti, and M. Reiher, Phys. Rev. A 83, 012508 (2011).

${ }^{7}$ L. K. McKemmish, R. H. McKenzie, N. S. Hush, and J. R. Reimers, J. Chem. Phys. 135, 244110 (2011).

${ }^{8}$ K. Boguslawski, K. H. Marti, O. Legeza, and M. Reiher, J. Chem. Theory Comput. 8, 1970 (2012).

${ }^{9}$ K. Boguslawski, P. Tecmer, Ö. Legeza, and M. Reiher, J. Phys. Chem. Lett. 3, 3129 (2012).

${ }^{10}$ K. Boguslawski, P. Tecmer, G. Barcza, Ö. Legeza, and M. Reiher, J. Chem. Theory Comput. 9, 2959 (2013).

${ }^{11}$ Y. Kurashige, G. K.-L. Chan, and T. Yanai, Nat. Chem. 5, 660 (2013).

${ }^{12}$ E. Fertitta, B. Paulus, G. Barcza, and Ö. Legeza, Phys. Rev. B 90, 245129 (2014).

${ }^{13}$ C. Duperrouzel, P. Tecmer, K. Boguslawski, G. Barcza, Ö. Legeza, and P. W. Ayers, Chem. Phys. Lett. 621, 160 (2015)

${ }^{14}$ V. Murg, F. Verstraete, R. Schneider, P. R. Nagy, and Ö. Legeza, J. Chem. Theory Comput. 11, 1027 (2015).

${ }^{15}$ S. Knecht, Ö. Legeza, and M. Reiher, J. Chem. Phys. 140, 041101 (2014).

${ }^{16} \mathrm{~K}$. Boguslawski and P. Tecmer, Int. J. Quantum Chem. 115, 1289 (2015).

${ }^{17}$ S. Szalay, M. Pfeffer, V. Murg, G. Barcza, F. Verstraete, R. Schneider, and Ö. Legeza, Int. J. Quantum Chem. 115, 1342 (2015).

${ }^{18}$ L. Freitag, S. Knecht, S. F. Keller, M. G. Delcey, F. Aquilante, T. Bondo Pedersen, R. Lindh, M. Reiher, and L. Gonzalez, Phys. Chem. Chem. Phys. 17, 14383 (2015).

${ }^{19}$ Y. Zhao, K. Boguslawski, P. Tecmer, C. Duperrouzel, G. Barcza, Ö. Legeza, and P. W. Ayers, Theor. Chem. Acc. 134, 120 (2015).
${ }^{20}$ T. Szilvási, G. Barcza, and Ö. Legeza, e-print arXiv:1509.04241 (2015).

${ }^{21}$ M. Molina-Espíritu, R. O. Esquivel, S. López-Rosa, and J. S. Dehesa, J. Chem. Theory Comput. 11, 5144 (2015).

${ }^{22}$ C. Krumnow, L. Veis, O. Legeza, and J. Eisert, Phys. Rev. Lett. 117, 210402 (2016).

${ }^{23}$ C. J. Stein and M. Reiher, J. Chem. Theory Comput. 12, 1760 (2016).

${ }^{24}$ C. Stein and M. Reiher, Chimia 71, 170 (2017).

${ }^{25}$ A. Kovyrshin and M. Reiher, J. Chem. Phys. 147, 214111 (2017).

${ }^{26}$ S. Szalay, G. Barcza, T. Szilvási, L. Veis, and Ö. Legeza, Sci. Rep. 7, 2237 (2017).

${ }^{27}$ C. Stemmle, B. Paulus, and Ö. Legeza, Phys. Rev. A 97, 022505 (2018).

${ }^{28}$ Y. Kurashige and T. Yanai, J. Chem. Phys. 130, 234114 (2009).

${ }^{29}$ V. Murg, F. Verstraete, O. Legeza, and R. M. Noack, Phys. Rev. B 82, 205105 (2010).

${ }^{30}$ N. Nakatani and G. K.-L. Chan, J. Chem. Phys. 138, 134113 (2013).

${ }^{31}$ G. K.-L. Chan, A. Kesselman, N. Nakatani, Z. Li, and S. R. White, J. Chem. Phys. 145, 014102 (2016)

${ }^{32}$ S. Keller, M. Dolfi, M. Troyer, and M. Reiher, J. Chem. Phys. 143, 244118 (2015).

${ }^{33}$ S. Wouters and D. Van Neck, Eur. Phys. J. D 68, 272 (2014).

${ }^{34}$ K. Gunst, F. Verstraete, S. Wouters, Ö. Legeza, and D. V. Neck, J. Chem. Theory Comput. 14, 2026 (2018).

${ }^{35}$ S. R. White, Phys. Rev. Lett. 69, 2863 (1992).

${ }^{36}$ S. R. White, Phys. Rev. B 48, 10345 (1993).

${ }^{37}$ U. Schollwöck, Ann. Phys. 326, 96 (2011).

${ }^{38}$ F. M. Faulstich, M. Máté, A. Laestadius, M. A. Csirik, L. Veis, A. Antalik, J. Brabec, R. Schneider, J. Pittner, S. Kvaal, and Ö. Legeza, J. Chem. Theory Comput. 15, 2206 (2019).

${ }^{39}$ The number in parentheses denotes the number of hydrogen atoms. 
${ }^{40}$ S.-L. Chou, J.-I. Lo, Y.-C. Peng, M.-Y. Lin, H.-C. Lu, B.-M. Cheng, and J. F. Ogilvie, Chem. Sci. 6, 6872 (2015).

${ }^{41}$ M. Arrowsmith, H. Braunschweig, M. A. Celik, T. Dellermann, R. D. Dewhurst, W. C. Ewing, K. Hammond, T. Kramer, I. Krummenacher, J. Mies, K. Radacki, and J. K. Schuster, Nat. Chem. 8, 890 (2016).

${ }^{42}$ J. Brabec, J. Lang, M. Saitow, J. Pittner, F. Neese, and O. Demel, J. Chem. Theory Comput. 14, 1370 (2018).

${ }^{43}$ H. C. Longuet-Higgins, J. Chem. Soc. 1946, 139.

${ }^{44}$ H. C. Longuet-Higgins and R. P. Bell, J. Chem. Soc. 1943, 250.

${ }^{45}$ W. H. Eberhardt, B. Crawford, and W. N. Lipscomb, J. Chem. Phys. 22, 989 (1954).

${ }^{46}$ K. Lammertsma and T. Ohwada, J. Am. Chem. Soc. 118, 7247 (1996).

${ }^{47}$ W. N. Lipscomb, Acc. Chem. Res. 6, 257 (1973).

${ }^{48}$ E. C. Neeve, S. J. Geier, I. A. I. Mkhalid, S. A. Westcott, and T. B. Marder, Chem. Rev. 116, 9091 (2016).

${ }^{49}$ M. A. Vincent and H. F. Schaefer, J. Am. Chem. Soc. 103, 5677 (1981).

${ }^{50}$ R. R. Mohr and W. N. Lipscomb, Inorg. Chem. 25, 1053 (1986).

${ }^{51}$ L. A. Curtiss and J. A. Pople, J. Chem. Phys. 90, 4314 (1989).

${ }^{52}$ L. A. Curtiss and J. A. Pople, J. Chem. Phys. 91, 5118 (1989).

${ }^{53}$ I. Demachy and F. Volatron, J. Phys. Chem. 98, 10728 (1994).

${ }^{54}$ I. Alkorta, I. Soteras, J. Elguero, and J. E. D. Bene, Phys. Chem. Chem. Phys. 13, 14026 (2011).

${ }^{55}$ P. P. Power, Nature 463, 171 (2010).

${ }^{56}$ P. P. Power, Chem. Rec. 12, 238 (2011).

${ }^{57}$ N. A. Giffin and J. D. Masuda, Coord. Chem. Rev. 255, 1342 (2011).

${ }^{58}$ M. Niemeyer and P. P. Power, Inorg. Chem. 36, 4688 (1997).

${ }^{59}$ D. Naglav, A. Neumann, D. Bläser, C. Wölper, R. Haack, G. Jansen, and S. Schulz, Chem. Commun. 51, 3889 (2015).

${ }^{60}$ T. Arnold, H. Braunschweig, W. C. Ewing, T. Kramer, J. Mies, and J. K. Schuster, Chem. Commun. 51, 737 (2015).

${ }^{61}$ H.-W. Lerner, S. Scholz, M. Bolte, N. Wiberg, H. Nöth, and I. Krossing, Eur. J. Inorg. Chem. 2003, 666.

${ }^{62}$ K. C. Mondal, H. W. Roesky, M. C. Schwarzer, G. Frenking, B. Niepötter, H. Wolf, R. Herbst-Irmer, and D. Stalke, Angew. Chem., Int. Ed. 52, 2963 (2013).

${ }^{63}$ Y. Li, K. C. Mondal, H. W. Roesky, H. Zhu, P. Stollberg, R. Herbst-Irmer, D. Stalke, and D. M. Andrada, J. Am. Chem. Soc. 135, 12422 (2013).

${ }^{64}$ S. Szalay, Phys. Rev. A 92, 042329 (2015).

${ }^{65} \mathrm{M}$. Ohya and D. Petz, Quantum Entropy and Its Use, 1st ed. (Springer-Verlag, 1993).

${ }^{66}$ H. Araki and H. Moriya, Rev. Math. Phys. 15, 93 (2003).

${ }^{67}$ M. M. Wilde, Quantum Information Theory (Cambridge University Press, 2013).

${ }^{68}$ R. Horodecki, P. Horodecki, M. Horodecki, and K. Horodecki, Rev. Mod. Phys. 81, 865 (2009).

${ }^{69}$ E. Schrödinger, Math. Proc. Cambridge Philos. Soc. 32, 446 (1936).

${ }^{70}$ L. P. Hughston, R. Jozsa, and W. K. Wootters, Phys. Lett. A 183, 14 (1993).
${ }^{71}$ B. A. Davey and H. A. Priestley, Introduction to Lattices and Order, 2nd ed. (Cambridge University Press, 2002).

${ }^{72}$ S. Szalay and Z. Kökényesi, Phys. Rev. A 86, 032341 (2012).

${ }^{73}$ S. Szalay, J. Phys. A: Math. Theor. 51, 485302 (2018).

${ }^{74}$ G. Adesso, T. R. Bromley, and M. Cianciaruso, J. Phys. A: Math. Theor. 49, 473001 (2016).

${ }^{75}$ Ö. Legeza and J. Sólyom, Phys. Rev. Lett. 96, 116401 (2006).

${ }^{76}$ R. F. Nalewajski, J. Phys. Chem. A 104, 11940 (2000).

${ }^{77}$ M. Mottet, P. Tecmer, K. Boguslawski, Ö. Legeza, and M. Reiher, Phys. Chem. Chem. Phys. 16, 8872 (2014).

${ }^{78}$ R. F. Nalewajski and E. Switka, Phys. Chem. Chem. Phys. 4, 4952 (2004).

${ }^{79}$ G. Barcza, R. M. Noack, J. Sólyom, and Ö. Legeza, Phys. Rev. B 92, 125140 (2015).

${ }^{80}$ G. Lindblad, Commun. Math. Phys. 33, 305 (1973).

${ }^{81}$ R. Horodecki, Phys. Lett. A 187, 145 (1994).

${ }^{82}$ Ö. Legeza, F. Gebhard, and J. Rissler, Phys. Rev. B 74, 195112 (2006).

${ }^{83}$ F. Herbut, J. Phys. A: Math. Gen. 37, 3535 (2004).

${ }^{84}$ K. Modi, T. Paterek, W. Son, V. Vedral, and M. Williamson, Phys. Rev. Lett. 104, 080501 (2010).

${ }^{85}$ C. H. Bennett, H. J. Bernstein, S. Popescu, and B. Schumacher, Phys. Rev. A 53, 2046 (1996).

${ }^{86} \mathrm{M}$. A. Nielsen and I. L. Chuang, Quantum Computation and Quantum Information, 1st ed. (Cambridge University Press, 2000).

${ }^{87}$ E. Schmidt, Math. Ann. 63, 433 (1907).

${ }^{88}$ J. Pipek and P. G. Mezey, J. Chem. Phys. 90, 4916 (1989).

${ }^{89}$ I. Mayer, Chem. Phys. Lett. 97, 270 (1983).

${ }^{90}$ I. Mayer, J. Comput. Chem. 28, 204 (2007).

${ }^{91}$ I. Mayer, Bond Orders and Energy Components: Extracting Chemical Information From Molecular Wave Functions (Taylor \& Francis, 2016).

${ }^{92}$ M. Kállay, Z. Rolik, J. Csontos, I. Ladjánszki, L. Szegedy, B. Ladóczki, G. Samu, K. Petrov, M. Farkas, P. Nagy, D. Mester, and B. Hégely, MRCC, a quantum chemical program suite, version 2016-07-15, 2016, see https://www.mrcc.hu/.

${ }^{93}$ Z. Rolik, L. Szegedy, I. Ladjánszki, B. Ladóczki, and M. Kállay, J. Chem. Phys. 139, 094105 (2013).

${ }^{94}$ D. Mester, J. Csontos, and M. Kállay, Theor. Chem. Acc. 134, 74 (2015).

${ }^{95}$ H. J. Werner, P. J. Knowles, G. Knizia, F. R. Manby, and M. Schütz, моцpro, version 2010.1, a package of $a b$ initio programs, 2010, see http://www.molpro.net.

${ }^{96}$ Ö. Legeza, L. Veis, and T. Mosoni, QC-DMRG-Budapest, a program for quantum chemical DMRG calculations.

${ }^{97}$ J. Chalupsky, "Charmol: Program for molecular graphics," https://sourceforge. net/projects/charmol, accessed 09 September 2018.

${ }^{98}$ Ö. Legeza, J. Röder, and B. A. Hess, Phys. Rev. B 67, 125114 (2003).

${ }^{99}$ T. J. Osborne and F. Verstraete, Phys. Rev. Lett. 96, 220503 (2006).

${ }^{100}$ V. Coffman, J. Kundu, and W. K. Wootters, Phys. Rev. A 61, 052306 (2000).

${ }^{101}$ A. Krapp, K. K. Pandey, and G. Frenking, J. Am. Chem. Soc. 129, 7596 (2007). 